\title{
Dynamic polygonal spreading of a droplet on a lyophilic pillar-arrayed surface
}

\author{
Enhui Chen ${ }^{\mathrm{a}, \mathrm{b}}$, Quanzi Yuana, Xianfu Huang ${ }^{\mathrm{a}}$ and Ya-Pu Zhao ${ }^{\mathrm{a}}$ \\ aState Key Laboratory of Nonlinear Mechanics, Institute of Mechanics, Chinese Academy of Sciences, Beijing \\ 100190, China; b University of Chinese Academy of Sciences, Beijing 100049, China
}

\begin{abstract}
We experimentally investigated the dynamic polygonal spreading of droplets on lyophilic pillar-arrayed substrates. When deposited on lyophilic rough surfaces, droplets adopt dynamic evolutions of projected shapes from initial circles to final bilayer polygons. These dynamic processes are distinguished in two regimes on the varied substrates. The bilayer structure of a droplet, induced by micropillars on the surface, was explained by the interaction between the fringe (liquid in the space among the micropillars) and the bulk (upper liquid). The evolution of polygonal shapes, following the symmetry of the pillar-arrayed surface, was analysed by the competition effects of excess driving energy and resistance which were induced by micropillars with increasing solid surface area fraction. Though the anisotropic droplets spread in different regimes, they obey the same scaling law $S \sim t^{2 / 3}$ ( $S$ being the wetted area and $t$ being the spreading time), which is derived from the molecular kinetic theory. These results may expand our knowledge of the liquid dynamics on patterned surfaces and assist surface design in practical applications.
\end{abstract}

\section{ARTICLE HISTORY}

Received 29 January 2016

Revised 12 April 2016

Accepted 13 April 2016

\section{KEYWORDS}

droplet; spreading; pattern; dynamic; contact line

\section{Introduction}

Spreading of a droplet on a micro-structured surface is prevalent in nature, $[1,2]$ and is of key importance in a wide range of applications, such as DNA technologies, [3,4] fog-harvesting, $[5,6]$ inkjet printing, $[7-9]$ biomedicine $[10,11]$ and microfluidics. [12,13] To develop these practical applications, dynamic wetting behaviours of a droplet on a textured surface have been investigated extensively in recent years. Compared to a smooth solid surface, the topography induces extensive specific effects to the spreading behaviours.[14-18] The moving contact line (MCL) becomes a complex curved line and propagates in a special stepwise mode. $[19,20]$ The scaling laws for the dynamic contact angles and spreading radius are modified.[1,21] Moreover, the shape of a droplet may spread into polygonal bilayer structure, instead of a simple spherical cap.

CONTACT Ya-PuZhao yzhao@imech.ac.cn

(-) Supplemental data for this article can be accessed here. 
When a droplet is released on a lyophilic pillar-arrayed surface, the base of the liquid penetrates into the space among the pillars, forming a fringe film. The upper part of the droplet collapses on the base of the fringe film, named the bulk. Time series of this process is illustrated in Figure 1. Wetting of a droplet on microstructured surface has been investigated intensely for decades. Extrand and cowokers [22] elegantly reported that appropriate lyophilic pillar arrays could effectively drive partial wetting liquids to complete wetting. Later, by varying the geometry of the surface array and the liquid, Courbin et al. [20] found a diversity of final wetted shapes, including polygons and circles, of droplets on pillar-arrayed surfaces. Raj et al. [23] then showed complete control of polygonal wetted shapes via the design of topographic or chemical heterogeneity on the surface. Jokinen et al. [24] developed a method of fabricating irregular pillars on a square array surface, and found directional wetting property of droplets on this surface, where droplets spread to irregular square-like shapes. and Vrancken et al. [7] reported the droplet shapes depend on the array geometry, pillar shape and array space. In previous studies, the researchers focused on the final shapes of droplets on lyophilic pillar-arrayed surfaces, while the evolution of the projected shape from an initial circle to a final polygon is essentially a dynamic process. Since the bulk liquid penetrates into the pillars progressively to supply the fringe propagation, the two parts may propagate with different shapes. Thus, a study of the dynamic spreading is necessary. Courbin et al. [25] reported the dynamics of shape evolution, while the mechanism in the process of evolution is still demanded. Kim and coworkers [26] quantified the dynamics of polygonal spreading and proposed the scaling laws for spreading rates of the bulk and the fringe separately, when the shape evolution of the bilayer structure has not been investigated yet.

In this work, we considered the dynamic polygonal spreading of a droplet on a lyophilic pillar-arrayed surface. Deposited on a lyophilic pillar-arrayed surface, the droplet spread with transient projected shapes from an initial singular circle, through evolving polygonal bulk and fringe, to an equilibrium shape. We demonstrated that the combined effect of the interfacial tension and the rough surface make the shape polygonal and bilayer structured. Then, we theoretically analysed the scaling law of the anisotropic dynamic spreading based on the molecular kinetic theory (MKT). Although the droplets spread in distinct patterns, they obeyed the same scaling law derived by MKT. Our work may help understand the mechanism of polygonal droplets on patterned surfaces, and design surface textures in practical applications.

\section{Experiments}

In our experiments, one ethanol droplet with radius of about $0.4 \mathrm{~mm}$ was produced and deposited on the pillar-arrayed Polydimethylsiloxane (PDMS) surface using a micropipette (Figure 2). Since the manipulation of the droplet requires large space above the transparent substrate, an inverted microscope is needed. Since the droplet spreads rapidly on the lyophilic PDMS substrate (the advancing, receding and equilibrium contact angles of an ethanol droplet on a smooth PDMS surface are measured about $33^{\circ}, 28^{\circ}$ and $30^{\circ}$, respectively), a high-speed camera is needed to capture the dynamic spreading process. The high-speed camera (HotShot 512 sc, NAC) was connected to the inverted microscope 


\section{(a)}
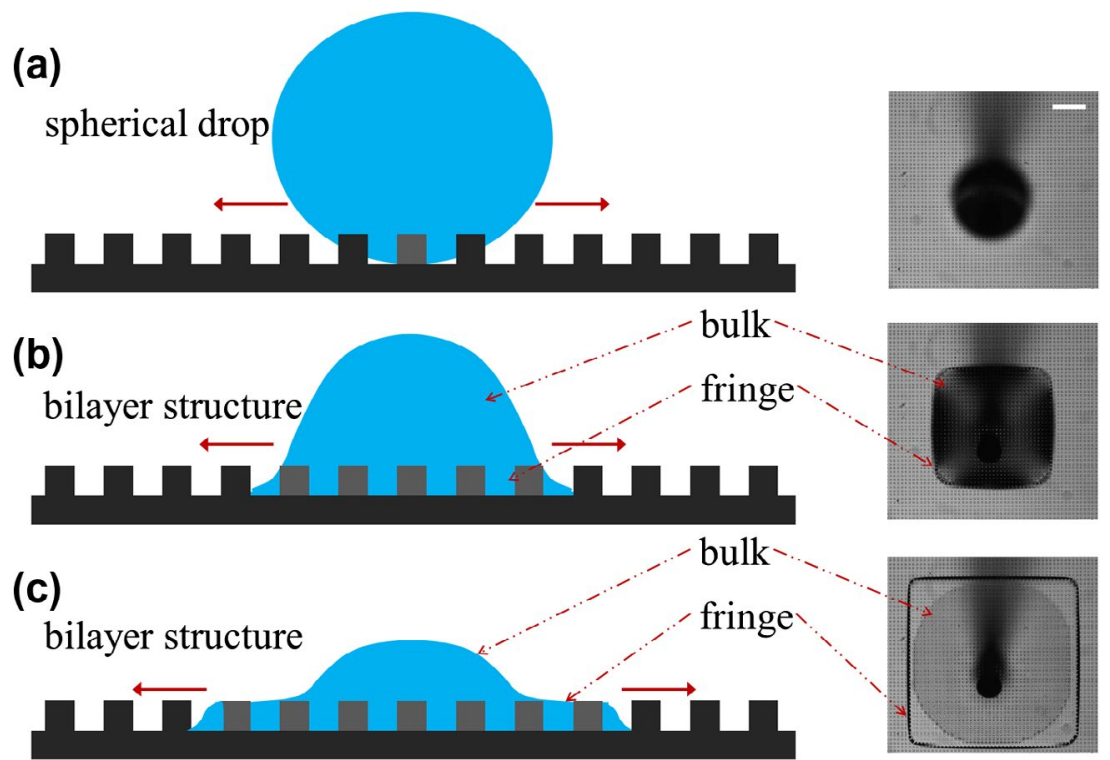

(d)

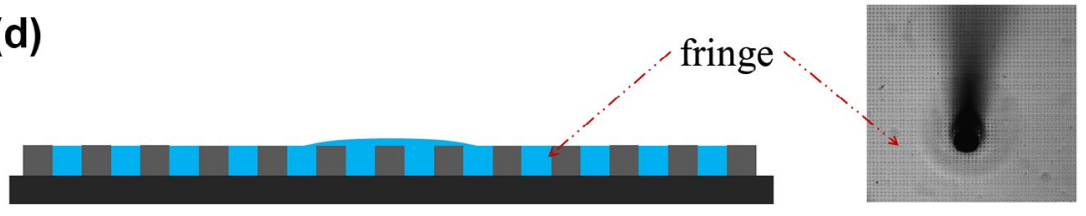

Figure 1. Time series showing the spreading process of a droplet on a lyophilic pillar-arrayed surface, side view (schematics) on the left and bottom view (snapshots) on the right, from an initial spherical droplet to a final liquid film. (a) An initial spherical droplet is deposited on the substrate. (b-d) The base of the droplet penetrates into the space among the pillars and forms the fringe. The liquid above the micropillars is called the bulk. The droplet propagates as a bilayer structure with the bulk and the fringe. The volume of the bulk shrinks progressively to supply the fringe in the dynamic spreading. (b) The droplet collapses to a spherical cap. (c) The contours of the bulk and the fringe separate gradually. The solid boundary in snapshot is the microscopic image of the fringe boundary. (d) The bulk shrinks until totally loss into the space among the pillars. Scale bar, $250 \mu \mathrm{m}$.

(IX71, Olympus) beneath the specimen platform. The entire experimental equipment was placed on the vibration damping platform.

The PDMS substrates with micropillar arrays fabricated on surface were made in two steps. First, the negative patterns were fabricated on silicon wafers using photolithography followed by a deep reactive ion etching process in the Institute of Microelectronics, Peking University. Then, the silicon negative patterns were used as moulds for patterning the PDMS via a series step of spin coating, curing and peeling. In the second step, the patterned silicon wafers were prepared with cleaning and silanization to facilitate the release of the elastomer PDMS from the wafers after curing. Liquid silicone prepolymer PDMS (Sylgard 184, Dow Corning) was compounded with the mass ratio of the base to crosslink 10:1. After being sufficiently stirred, the liquid PDMS was poured on the wafers and spin coated at 300 r.p.m. for $30 \mathrm{~s}$ using a spin coater (MODEL WS-400BZ-6NPP/LITE, Laurell). Then, the silicon wafers together with PDMS films were degased for $20 \mathrm{~min}$ and cured at $80^{\circ} \mathrm{C}$ for $6 \mathrm{~h}$. Finally, the PDMS films were peeled off from the silicon wafers. After the above 


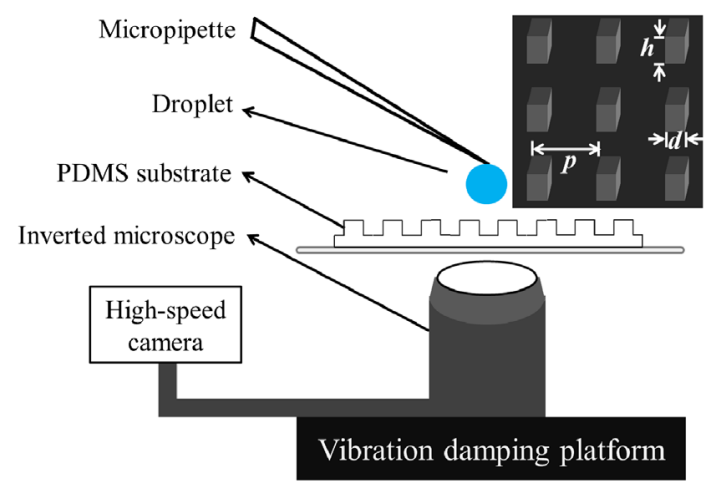

Figure 2. Schematic of the experimental set-up. Inset: schematic of the PDMS pillar-arrayed surface. $d, p$ and $h$ are the size, the period and the height of the micro pillars, respectively; $[d, p, h]$ of the substrates used in the experiments are listed in Table 1.

Table 1. Substrates of different parameters used in the experiments.

\begin{tabular}{lccccc}
\hline Sample & 1 & 2 & 3 & 4 & 5 \\
\hline$d(\mu \mathrm{m})$ & 5 & 5 & 10 & 15 & 15 \\
$p(\mu \mathrm{m})$ & 25 & 20 & 30 & 35 & 20 \\
$h(\mu \mathrm{m})$ & 30 & 30 & 30 & 30 & 30 \\
$s(\mu \mathrm{m})$ & 20 & 15 & 20 & 20 & 5 \\
$r o=1+4 d h / p^{2}$ & 1.96 & 2.50 & 2.33 & 2.47 & 5.50 \\
$\phi_{s}=d^{2} / p^{2}$ & 0.04 & 0.06 & 0.11 & 0.18 & 0.56 \\
\hline
\end{tabular}

process, PDMS substrates with micropillar arrays fabricated on surface were prepared for the dynamic wetting experiments.

The experiments were carried on different pillar-arrayed PDMS substrates with varied topological parameters $[d, p, h]$, The experiments were carried on different pillar-arrayed PDMS substrates with varied topological parameters $[d, p, h]$, where $d, p$ and $h$ are the side length, period and height of the square pillars, respectively (inserted in Figure 2 ). The spacing between pillars $s$ is obtained $s=p-d$. Solid surface area fraction $\phi_{\mathrm{s}}=d^{2} / p^{2}$ and surface roughness (ratio of actual solid area to projected solid area) ro $=1+4 d h / p^{2}$. These parameters for each sample were listed in Table 1.

The ethanol droplets used in the experiments have volume $V \sim 0.3 \mu \mathrm{L}$, density $\rho=789$ $\mathrm{kg} \mathrm{m}^{-3}$, viscosity $\mu=0.0012 \mathrm{~Pa} \cdot \mathrm{s}$, surface tension $\gamma_{\mathrm{LV}}=0.022 \mathrm{~N} \mathrm{~m}^{-1}$ and capillary length $l_{\mathrm{CA}}=\sqrt{\gamma_{\mathrm{LV}} /(\rho g)} \sim 1.69 \mathrm{~mm}$.

\section{Results and discussions}

\subsection{Experimental results}

In our experiments, when deposited on different lyophilic pillar-arrayed surfaces, ethanol droplets exhibited two distinct spreading regimes, as shown in Figure 3: (1) in the case of low $\phi_{\mathrm{s}}$ samples (Samples 1 and 2 in Table 1 ), the wetted area of the spreading droplet developed from an initial circle to an octagonal shape. The bulk and the fringe shared the same outline when spreading (electronic supplementary material, video S1). (2) in the case of high $\phi_{s}$ 


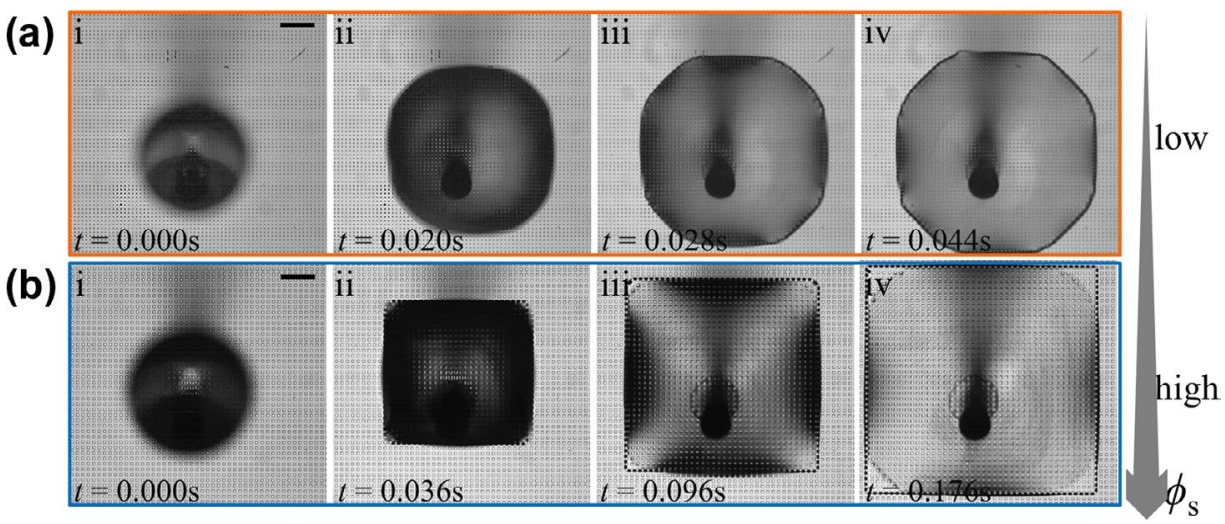

Figure 3. The two dynamic spreading regimes on (a) low $\phi_{\mathrm{s}}$ sample and (b) high $\phi_{\mathrm{s}}$ sample, respectively. Scale bar, $200 \mu \mathrm{m}$.

samples (Samples 3, 4 and 5 in Table 1), the wetted area of the spreading droplet developed from an initial circle, through a square shape, separated to a bilayer structure, and ended up with a rounded octagonal bulk and a square fringe (electronic supplementary material, video S2). In these regimes, spreading times taken into consideration are less than $0.6 \%$ of the duration of spreading experiments (ranging from 30 to $120 \mathrm{~s}$ for different samples). So we neglected the effect of evaporation to shape evolution. All these spreading behaviours are repeatable.

In these two spreading regimes, there was a remarkable phenomenon to mention: the shade distribution of the wetted area was anisotropic, similar to the anisotropic spreading droplet. The dark area, which was caused by the inclined liquid-vapour interface,[19] increased with the distance to the centre of the wetted region. Moreover, the dark area along the axes was obviously larger than along the diagonal, indicating the existence of the thickness gradient of the bulk. In these observations, one initial spherical droplet on homogeneous surface adopted anisotropic spreading and evolved to a bilayer structure.

\subsection{Fringe shapes}

To explain these phenomena, it is essential to understand the multiple driving force and resistance that act on the liquid in the dynamic spreading process. There are several forces that conduct the spreading behaviour: gravity, interfacial tension $\gamma$, viscosity $\mu$, and inertia.[19] Since the radius of the droplet is much smaller than the capillary length of the ethanol $(1.69 \mathrm{~mm})$, the interfacial tension takes priority over gravity. The Weber number $W e=\rho v^{2} l / \gamma_{\mathrm{LV}}$ ( $v$ is the velocity of the MCL, $l$ is the droplet diameter), which represents the ratio of inertia to the surface tension, is about 0.2 , much less than the threshold 1.1, indicating the interfacial tension takes priority over the inertia.[27] Hence, the main driving force for the droplet is the interfacial tension.

Furthermore, the forest of lyophilic pillars induces two effects to the droplet. On the one hand, the pillars introduce obstacles and excess resistance to the fringe. On the other hand, the excess lyophilic solid surface provides extra driving force to the MCL, inducing the fringe liquid to accelerate at the interior corners between pillars 
and substrate, which is known as the Concus-Finn effect.[28] These two effects compete with each other.[19]

The interfacial tension and the driving effects from micropillars generate the polygonal-shaped fringe. Driven by interfacial tension, the liquid tends to adopt a spherical cap shape. However, the excess driving force and the interior corners force the fringe to a square shape, which corresponds to the symmetry of the patterned surface, as shown in the right side of Figure 4(a). We adopted the solid area fraction $\phi_{s}$ to characterize excess driving force and the interior corner acceleration. Thus, a circular fringe when $\phi_{s}$ approaches 0 (a smooth surface) and a square fringe when $\phi_{s}$ is large enough could be deduced. An intermediate shape of the fringe film is expected between a spherical cap and a square, as the effects of both excess driving force and acceleration of interior corner varied with $\phi_{s}$. Considering the symmetry of a circle and a square, the intermediate shape could be inferred an octagon (left side in Figure 4(a)). These predictions were consistent with the experimental results: the octagonal fringe in the low $\phi_{s}$ regime, and the square fringe in the high $\phi_{s}$ regime.

\subsection{Bulk shapes}

Spreading on the fringe, the front of the bulk actually propagates on liquid film. How could the bulk adopt an octagon or rounded octagon shape? Firstly, the bulk is driven merely by surface tension rather than excess driving force from micropillars. So the bulk prefers to adopt a circular shape. Secondly, the bulk shall not propagate beyond the fringe border.

Once an initial droplet contacts with the lyophilic solid surface, it collapses to a spherical cap, and the bottom liquid penetrates into the pillars quickly and forms the fringe. The fringe expands very rapidly, while the bulk liquid is strictly restricted within the fringe border. As the result, the bulk spreads together with the square or octagonal fringe in the beginning.

In the next stage of propagation, we come to the high $\phi_{s}$ regime first. In this regime, the fringe advances faster than the bulk owing to the excess driving effects. As a result, the bulk separates from the fringe gradually in the early stage of spreading ( $\sim 0.5 \mathrm{~s}$ from spreading). The mechanism of the rounded-octagonal bulk was illustrated in Figure 4(c) (iii). Suppose a square expand from the centre of a circle. With the side length of the square increasing, the four corners of the square first go beyond the circular area. On the opposite side, the four bows of the circle are beyond the square area. Take the bulk (cyan) as a circle and the fringe (grey) as a square, then two conclusions can be made. First, with the growth of the fringe, the separation between the fringe and the bulk begins in the diagonal directions. Second, the bulk is restricted by the square fringe in the axial directions. Owing to these two conclusions, the rounded octagonal bulk (cyan area) yields. This shape explanation agrees with the experimental result.

In the case of the low $\phi_{s}$ regime, we found the separation of the bulk from the fringe much more difficult than in the high $\phi_{s}$ regime. The effects induced by low $\phi_{s}$ are the main factors. Low $\phi_{s}(\sim 0.0100)$ indicates low excess driving force and weak interior corner acceleration. As mentioned above, micropillars also induce resistance to the spreading. Here, the pinning effect of the MCL characterized by aspect ratio $h /(p-d)$ [20] brings in the resistance. To the sample with lowest $\phi_{\mathrm{s}}(=0.0400)$, the low excess driving force is offset by the pinning effect, resulting in no separation between the bulk and the fringe in the experimental observation. To the sample with the second lowest $\phi_{s}(=0.0625)$, the excess driving force slightly overcomes the pinning effect, hence the experimental observation of mild separation in 
(a)

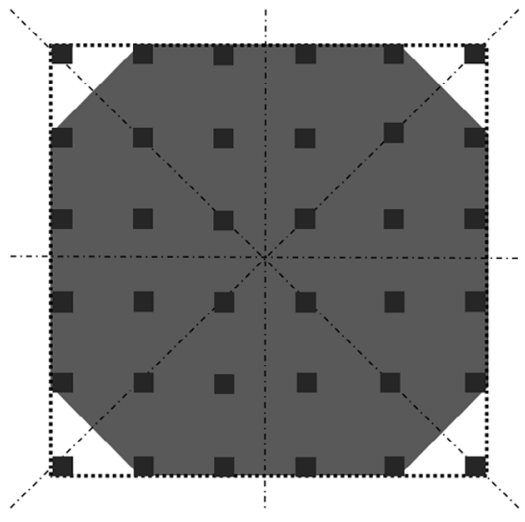

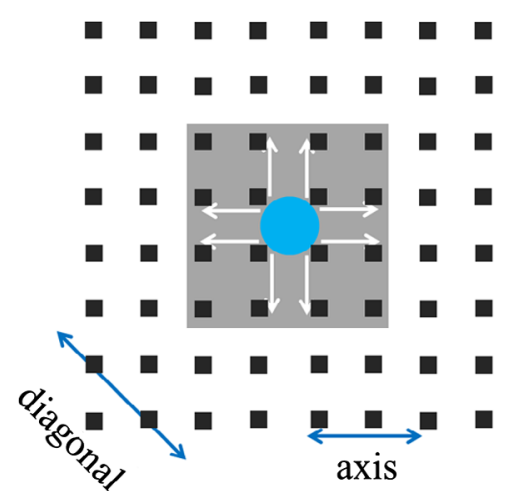

(b)

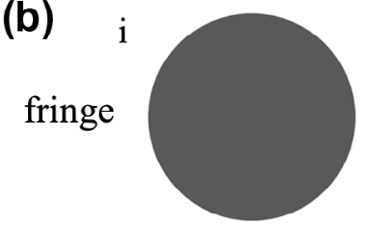

ii

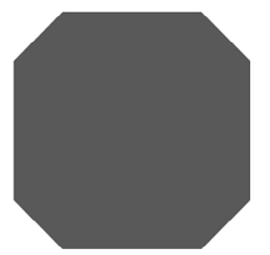

iii

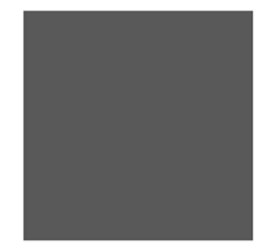

(c)

$$
0
$$
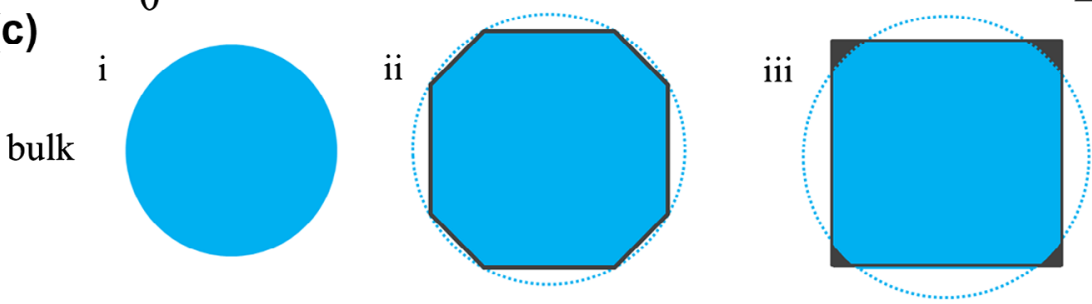

Figure 4. Schematics of droplet shapes. (a) Schematics of the octagonal fringe (left) and the square fringe (right). The black dices represent the pillar arrays. Left side in (b), under the driving force from the substrate (the white arrays), the initial droplet (the cyan circle) propagate to a square fringe (the grey square). (b-c) Shape evolution of (b) fringe, and (c) bulk with respect to the solid surface area fraction $\phi_{s}$. In (c), the cyan regions represent the bulk, dotted cyan circles represent supposed bulk, and grey regions represent the fringe.

the later stage of spreading (at $4.824 \mathrm{~s}$ from spreading) can be understood. As illustrated in Figure 4(c) (ii), the bulk is restricted to the octagonal fringe both in the axial and diagonal directions. For this reason, the bulk propagates together with the fringe to an octagonal shape (cyan region), which agrees with the experimental result.

\subsection{Shade distributions and surface feature simulations}

Our explanations were validated by the distribution characteristics of shade. The shade is caused by the inclined liquid-vapour interface (Figure 5(a)), and the inclined liquid-vapour interface is generated necessarily by the restriction of the bulk liquid. The bows in Figure 4(c) (ii) and (iii) represent the restricted areas of the bulk. And these bows were found corresponding to the dark areas in the experiments: (1) the areas of bows along the 
(a)

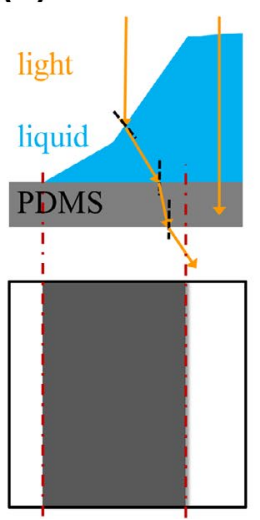

(b)
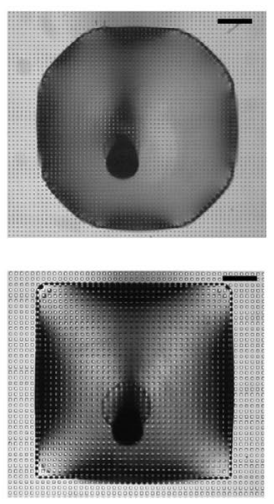

(c)

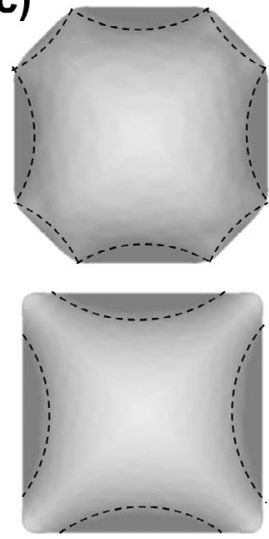

(d)
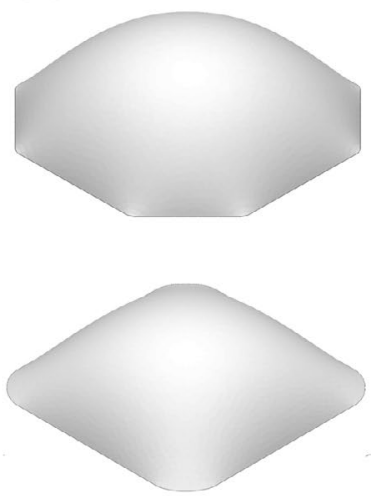

Figure 5. The shade distributions of propagating bulks. (a) Schematics of the inclined surface (upper) corresponding to the shade (lower). The refraction makes the inverted microscope (beneath the substrate) receive less light from the inclined interface than smooth interface. Thus, the shade yields. (b-d) Shade distributions in (b) snapshots, (c) 2-dimensional modelling results, (d) 3-dimensional modelling results. The dashed circular arcs labelled in (c) aim to help distinguish the shade from the bright area. Scale bar, $200 \mu \mathrm{m}$.

diagonal are smaller than along the axes, corresponding to the experiments results in the low $\phi_{s}$ regime; also the dark area decreases with the restriction relaxing gradually in the spreading process. (2) In Figure 3(b), (ii) the bulk is restricted to the square, causing broad dark region along the border, (iii) along the diagonals the bulk is relaxed, while along the axes the bulk is restricted, resulting in light area along the former direction and dark area along the latter direction, (iv) with further spreading, the restriction of the bulk along the axes was relaxed more, resulting in only small dark region in the axial border.

By using finiteelement method (FEM), we also simulated the surface feature for bulk droplets limited within an octagonal fringe and a square fringe, and 3-dimensional renderings of bulks (Figure 5(c)-(d)), respectively.[29] The FEM simulations were carried out employing the Surface Evolver to achieve an equilibrium shape of a droplet on the pillar-arrayed surfaces [30]. Surface Evolver is a program that computes the minimal energy shape of a surface under constraints. The energy includes surface energies, gravity, and the converted constrains. In our situations, when deposited on the solid surface, the bulk droplet was driven to spread by surface tension. The equilibrium contact angle of the liquid on the solid was about $30^{\circ}$, obtained from the experiments. As discussed in previous section, gravity can be neglected, and the bulk was restricted by the fringe. This restriction was expressed by edge constraints in the simulations. The evaporation can be neglected in the early stage of spreading, so the liquid volume is kept constant. We adopted very fine mesh resolution and set the initial edge length less than $0.02 R_{\mathrm{b}}\left(R_{\mathrm{b}}\right.$ is the initial droplet radius) in each performance. The liquid surface evolved towards a minimal energy by a gradient descent method and achieved an equilibrium shape when the total energy achieved a balance between the surface tension and the restriction. The shade distributions in the modelling results agreed well with our experimental observations and explanations. 


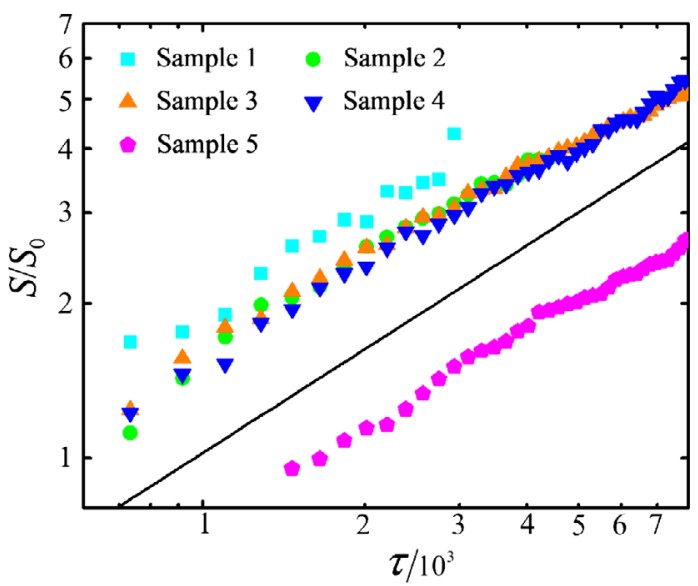

Figure 6. Evolution of wetted area with respect to time. $S / S_{0}$ is the dimensionless wetted area, $\tau$ is the dimensionless time. The black solid line represents the scaling law $S / S_{0} \sim \tau^{2 / 3}$.

\subsection{Scaling analysis by MKT}

To reveal the physical mechanism of a spreading droplet, we adopted MKT to carry out the scaling analysis of liquid propagation on a lyophilic surface.[19] The MKT is first proposed by Glasstone, Laidler \& Eyring, who view the liquid motion as a stress-modified molecular rate process.[31] This theory assumes that the fluids are mutually saturated, and on the solid surface, there are plenty of identical sites where liquid molecules could be adsorbed and desorbed. Considering a liquid incompletely wet, the solid, the adsorption at the solid-liquid interface differs from that at the solid-vapour interface. [32,33] According to MKT, the statistical dynamics of molecules at the three-phase region determines the motion of the MCL.

When liquid spreads over a solid surface, the solid surface adsorbs the liquid molecules, while the liquid molecules desorb and tend to advance. The adsorption and the desorption cause the energy dissipation. In the equilibrium state, the advancing frequency of liquid molecules $\kappa^{+}$and the receding frequency $\kappa^{-}$equal to the equilibrium frequency $\kappa_{0}$, leading to a static contact line:

$$
\kappa_{0}=\frac{k_{\mathrm{B}} T}{\mu v_{\mathrm{m}}} \exp \left(-\frac{\lambda^{2} W a}{k_{\mathrm{B}} T}\right),
$$

where $k_{\mathrm{B}}, T, \mu, v_{\mathrm{m}}, \lambda$ and $W a$ are the Boltzmann constant, absolute temperature, liquid viscosity, molecular flow volume, the spacing of surface sites and adhesion work between solid and liquid, respectively. Once applied, a driving force to the liquid molecules, the potential surface tilts, and the equilibrium state is disturbed. Modified by the driving work per unit area $w$, the advancing frequency and receding frequency are respectively,

$$
\begin{gathered}
\kappa^{+}=\kappa_{0} \exp \left(\frac{w \lambda^{2}}{2 k_{\mathrm{B}} T}\right), \\
\kappa^{-}=\kappa_{0} \exp \left(-\frac{w \lambda^{2}}{2 k_{\mathrm{B}} T}\right) .
\end{gathered}
$$


Here, the driving work per unit area $w$ equals to the interfacial energy change in liquid propagation $w=\left(\gamma_{\mathrm{SV}}-\gamma_{\mathrm{SL}}\right)$ ro $-\gamma_{\mathrm{LV}} \cos \theta$, where $\gamma_{\mathrm{SV}} \gamma_{\mathrm{SL}}$ and $\gamma_{\mathrm{LV}}$ are the solid-vapour, solid-liquid and liquid-vapour interface energies, respectively, $\theta$ is the instant contact angle, and surface roughness $r o=1+4 d h / p^{2}$. The value of $w \lambda^{2} / 2 k_{\mathrm{B}} T$ in our case is in the order of $0.01-0.1$, therefore $\sinh \left(w \lambda^{2} / 2 k_{\mathrm{B}} T\right) \sim w \lambda^{2} / 2 k_{\mathrm{B}} T$. The difference of the advancing and receding frequencies results the velocity of the MCL:

$$
v=\lambda\left(\kappa^{+}-\kappa^{-}\right)=2 \frac{k_{\mathrm{B}} T \lambda}{\mu v_{\mathrm{m}}} \exp \left(-\frac{\lambda^{2} W a}{k_{\mathrm{B}} T}\right) \sinh \frac{w \lambda^{2}}{2 k_{\mathrm{B}} T} \sim \frac{w \lambda^{3}}{\mu v_{\mathrm{m}}} \exp \left(-\frac{\lambda^{2} W a}{k_{\mathrm{B}} T}\right) .
$$

When the solid-liquid pair is fixed, $\lambda, v_{\mathrm{m}}$ and $W a$ become constants, $v \sim w / \mu$. Substituting Young's equation $\gamma_{\mathrm{SV}}-\gamma_{\mathrm{SL}}=\gamma_{\mathrm{LV}} \cos \theta_{\mathrm{eq}}$ [34] for $w, v \sim\left(\operatorname{rocos} \theta_{\mathrm{eq}}-\cos \theta\right) \gamma_{\mathrm{LV}} / \mu$ is obtained.

Considering the constant droplet volume (as liquid evaporation can be neglected in the early stage of propagation), $V_{0}=V_{\text {bulk }}+V_{\text {fringe }}$, that is:

$$
\frac{4}{3} \pi R_{0}^{3}=\frac{1}{6} \alpha H_{\mathrm{b}}\left(3 R_{\mathrm{b}}^{2}+H_{\mathrm{b}}^{2}\right)+\beta\left(1-\varphi_{\mathrm{s}}\right) h R_{\mathrm{f}}^{2},
$$

where $R_{0}, R_{\mathrm{b}}, H_{\mathrm{b}}$ and $R_{\mathrm{f}}$ are the radius of the initial spherical droplet, the radius of the bulk, the height of the bulk and the radius of the fringe, respectively; $\alpha, \beta$ are area coefficients for polygonal shapes, also independent of time. Take account of lubrication approximation $\left(H_{\mathrm{b}}<<R_{\mathrm{b}}\right.$ , $\left.\theta \sim H_{\mathrm{b}} / R_{\mathrm{b}}\right)$, [35] we get $v \sim \theta^{2} \gamma_{\mathrm{LV}} / \mu, \theta \sim H_{\mathrm{b}} / R_{\mathrm{b}} \sim\left(8 \pi R_{0}^{3}-6 \beta\left(1-\phi_{\mathrm{s}}\right) h R_{\mathrm{f}}^{2}\right) / 3 \alpha R_{\mathrm{b}}^{3}$. For rough surface in our case, $\theta$ could be simplify to $\theta \sim\left(1-\phi_{\mathrm{s}}\right) h R_{\mathrm{f}}^{2} / R_{\mathrm{b}}^{3}$ [19]. Considering $\varepsilon R_{\mathrm{b}} \sim R_{\mathrm{f}}$ ( $\varepsilon$ is independent of time), which is validated by previous work [26], the velocity could be expressed by

$$
v \sim \frac{\mathrm{d} R_{\mathrm{f}}}{\mathrm{d} t} \sim \frac{\gamma_{\mathrm{LV}} \varepsilon^{6}\left(1-\phi_{\mathrm{s}}\right)^{2} h^{2}}{\mu} \frac{1}{R_{\mathrm{f}}^{2}}
$$

Plug the instant wetted area $S \sim R_{\mathrm{f}}^{2}$ into Equation (6), and then the dimensionless solution $S / S_{0} \sim \tau^{2 / 3}$ is obtained, where $S_{0}$ is the initial projected area, $S / S_{0}$ is the dimensionless wetted area, and $\tau=t \gamma_{\mathrm{LV}} / \mu R_{0}$ is the dimensionless time, respectively.

We compared the experiments with the MKT result, since the homogeneous PDMS surface and the ethanol liquid used in the experiments satisfy the assumptions of MKT. In our experiments, the evaporation could be neglected when considering the early stage of propagation (within $0.2 \mathrm{~s}$ in scaling analysis). Figure 6 shows the experimental results in logarithmic coordinates. The abscissa represents $\tau$ evolution, and the ordinate represents $S / S_{0}$. Although droplets on five samples follow different spreading regimes, they approximately obey the same scaling law $S / S_{0} \sim \tau^{2 / 3}$. The experiments are in good agreement with the MKT result, indicating that polygonal spreading follows the same scaling law with circular spreading.[19]

\section{Conclusions}

In this work, we investigated the dynamic polygonal spreading of a droplet on a lyophilic pillar-arrayed surface. 
Firstly, in the experiments with ethanol droplets on varied topological substrates, two dynamic spreading regimes have been distinguished. In these regimes, the projected shapes of droplets evolve from initial circles to bilayer polygons. Controlled by interfacial tension and driving energy from micropillars, which induce acceleration at the interior corners and excess driving force, the fringes follow the symmetry of patterned surface and exhibit circular, octagonal and square projected shapes.

Secondly, in the bilayer structure dynamics, the combined evolution and the separation of the bulk and the fringe were caused by the competition between the excess driving energy and resistance from the micropillars. These explanations were also validated by shade distributions in both experimental snapshots and modelling results.

Furthermore, the physical mechanism of the dynamic process was theoretically analysed using MKT. The anisotropic spreading in the experiments agree well with MKT scaling of $S / S_{0} \sim \tau^{2 / 3}$.

Our results may help in understanding the complex effects caused by the pillar-arrayed surface and increase the toolbox of yielding polygonal patterns by changing topological parameters of the substrate.

\section{Disclosure statement}

No potential conflict of interest was reported by the authors.

\section{Funding}

This work was jointly supported by the National Natural Science Foundation of China [NSFC, grant number U1562105], [grant number 11372313], [grant number 11611130019] and CAS Interdisciplinary Innovation Team Project.

\section{References}

[1] Zhao YP. Moving contact line problem: advances and perspectives. Theor. Appl. Mech. Lett. 2014;4:034002.

[2] Bonn D, Eggers J, Indekeu J, et al. Wetting and spreading. Rev. Mod. Phys. 2009;81:739-805.

[3] Blanchard AP, Kaiser RJ, Hood LE. High-density oligonucleotide arrays. Biosens. Bioelectron. 1996;11:687-690.

[4] McHale G, Shirtcliffe NJ, Aqil S, et al. Topography driven spreading. Phys. Rev. Lett. 2004;93:036102.

[5] Parker AR, Lawrence CR. Water capture by a desert beetle. Nature. 2001;414:33-34.

[6] Grosu G, Andrzejewski L, Veilleux G, et al. Relation between the size of fog droplets and their contact angles with CR39 surfaces. J. Phys. D: Appl. Phys. 2004;37:3350-3355.

[7] Vrancken RJ, Blow ML, Kusumaatmaja H, et al. Anisotropic wetting and de-wetting of drops on substrates patterned with polygonal posts. Soft Matter. 2013;9:674-683.

[8] Derby B. Inkjet printing of functional and structural materials: fluid property requirements, feature stability, and resolution. Annu. Rev. Mater. Res. 2010;40:395-414.

[9] Rozhkov A, Prunet-Foch B, Vignes-Adler M. Impact of drops of surfactant solutions on small targets. Proc. R. Soc. London, Ser. A. 2010;466:2897-2916.

[10] Nagrath S, Sequist LV, Maheswaran S, et al. Isolation of rare circulating tumour cells in cancer patients by microchip technology. Nature. 2007;450:1235-1239.

[11] Key I, Pina S, Agathopoulos S, et al. Interfacial interactions between liquid new biocompatible model glasses and solid metallic and ceramic substrates used in biomedicine. Key Eng. Mater. 2005;284-286:835-838. 
[12] Skelley AM, Kirak O, Suh H, et al. Microfluidic control of cell pairing and fusion. Nat. Methods. 2009;6:147-152.

[13] Wang H, Wang W, Li L, et al. Surface microfluidic patterning and transporting organic small molecules. Small. 2014;10:2549-2552.

[14] Wenzel RN. Resistance of solid surfaces to wetting by water. Ind. Eng. Chem. Res. 1936;28:988994.

[15] Sun W, Yang F. Fabrication of asymmetric-gradient-concentric ring patterns via evaporation of droplets of PMMA solution at different substrate temperatures. RSC Adv. 2015;5:29850-29858.

[16] McHale G, Rowan SM, Newton MI, et al. Estimation of contact angles on fibers. J. Adhes. Sci. Technol. 1999;13:1457-1469.

[17] Wang FC, Wu HA. Pinning and depinning mechanism of the contact line during evaporation of nano-droplets sessile on textured surfaces. Soft Matter. 2013;9:5703-5709.

[18] Yu YS. Circular plate deformed by a sessile droplet. J. Adhes. Sci. Technol. 2014;28:1970-1979.

[19] Yuan QZ, Zhao YP. Multiscale dynamic wetting of a droplet on a lyophilic pillar-arrayed surface. J. Fluid Mech. 2013;716:171-188.

[20] Courbin L, Denieul E, Dressaire E, et al. Imbibition by polygonal spreading on microdecorated surfaces. Nat. Mater. 2007;6:661-664.

[21] McHale G, Newton MI, Shirtcliffe NJ. Dynamic wetting and spreading and the role of topography. J. Phys. : Condens. Matter. 2009;21:464122.

[22] Extrand CW, Moon SI, Hall P, et al. Superwetting of structured surfaces. Langmuir. 2007;23:8882-8890.

[23] Raj R, Adera S, Enright R, et al. High-resolution liquid patterns via three-dimensional droplet shape control. Nat. Commun. 2014;5:4975.

[24] Jokinen V, Leinikka M, Franssila S. Microstructured surfaces for directional wetting. Adv. Mater. 2009;21:4835-4838.

[25] Courbin L, Bird JC, Reyssat M, et al. Dynamics of wetting: from inertial spreading to viscous imbibition. J. Phys. : Condens. Matter. 2009;21:464127.

[26] Kim SJ, Moon MW, Lee KR, et al. Liquid spreading on superhydrophilic micropillar arrays. J. Fluid Mech. 2011;680:477-487.

[27] Duan RQ, Koshizuka S, Oka Y. Two-dimensional simulation of drop deformation and breakup at around the critical Weber number. Nucl. Eng. Des. 2003;225:37-48.

[28] Concus P, Finn R. On the behavior of a capillary surface in a wedge. Proc. Nat. Acad. Sci. 1969;63:292-299.

[29] Brakke KA. The surface evolver. Exp. Math. 1992;1:141-165.

[30] Surface Evolver. In surface evolver. Available from: http://www.susqu.edu/brakke/evolver/ evolver.html

[31] Glasstone S, Laidler KJ, Eyring H. The theory of rate processes: The kinetics of chemical reactions, viscosity, diffusion and electrochemical phenomena. New York (NY): McGrawHill; 1941.

[32] Blake T, Haynes J. Kinetics of liquidliquid displacement. J. Colloid Interface Sci. 1969;30:421423.

[33] Blake TD. The physics of moving wetting lines. J. Colloid Interface Sci. 2006;299:1-13.

[34] Young T. An essay on the cohesion of fluids. Philos. Trans. R. Soc. London. 1805;95:65-87.

[35] Greenspan HP. On the motion of a small viscous droplet that wets a surface. J. Fluid Mech. 1978;84:125-143. 